\title{
Gastric Perforation in the Postpartum Revealing Trichobizoar: A Case Report and Literature Review

\author{
Fahd MOUSSAID ${ }^{1 *}$, Ibrahim OUBIHI ${ }^{1}$, Anas HEDAJI ${ }^{1}$, Houssam REBAHI ${ }^{1}$, Ahmed Ghassan EL ADIB ${ }^{1}$
}

\begin{abstract}
${ }^{1}$ Mother and Child Hospital, Mohammed VI University Hospital, Marrakech, Morocco
DOI: $10.36347 /$ sasjm.2021.v07i04.001

| Received: 03.03.2021 | Accepted: 20.03.2021 | Published: 06.04.2021

*Corresponding author: Fahd Moussaid

Abstract

Case Report

Trichobezoars are a collection of hair or hair-like material within the gastrointestinal tract Clinically asymptomatic. This observation describes trichobezoar illustrated by gastric perforation occurring in the post partum period. An 35years-old multiparous woman, she previously presented in active labour and spontaneaously give birth to a stillborn baby. There was no past of depression or anxiety. Three days later the woman presented to the emergency departement with a sudden onset of upper abdominal pain and vomiting. Clinically, she was throught to have peritonitis. Peroperative exploration reveals a large gastric perforation, the stomach was noted to be enlarged by a firm, uniform mass. This later was identified as a trichobezoar. Trichobezoar is a rare entity which generally presents insidiously with non-specific abdominal symptoms. After the exclusion of more common etiologies, trichobezoar should be considered in all young females presenting with symptoms of gastrointestinal obstruction.

Keywords: Trichobezoars - Post partum period - Perforation.

Copyright $\odot 2021$ The Author(s): This is an open-access article distributed under the terms of the Creative Commons Attribution 4.0 International License (CC BY-NC 4.0) which permits unrestricted use, distribution, and reproduction in any medium for non-commercial use provided the original author and source are credited.
\end{abstract}

\section{INTRODUCTION}

Gastric trichobezoars are an uncommon condition, usually seen in young women with a history of trichotillomania and trichophagy [1]. The term bezoar refers to various foreign bodies found in the gastrointestinal tract. Most of them are formed in the stomach by the accumulation of non-digestible substances, such as certain vegetable fibres (phytobezoar), hair (trichobezoar), concentrated dairy products (lactobezoar), more rarely certain drugs (pharmacobezoar) [2].

Trichobezoars are accumulations of hair or hair-like material within the gastrointestinal tract [3]. Clinically asymptomatic, its diagnosis is essentially based on fibroscopy and its treatment is essentially surgical [4].

This observation describes gastric perforation as a life-threatening complication of trichobezoar, in postpartum of normal vaginal delivery, and addresses pregnancy management in women with trichotillomania.

\section{CASE REPORT}

An 35-years-old multiparous woman, previously she presented in active labour and spontaneously give birth to a stillborn baby. There was no other relevant medical history. She denied any history of depression or anxiety. Three days later the woman presented to the emergency department with a $12 \mathrm{~h}$ history of sudden onset upper abdominal pain and vomiting. Clinically, she was through to have peritonitis, with epigastric masse. The remaining laboratory investigation showed haemoglobin $(\mathrm{Hb}) 12$ $\mathrm{g} / \mathrm{l}$, WCC 9450 per $\mathrm{mm}^{3}$, the reactive C protein 658 $\mathrm{mg} / \mathrm{l}$. Computed tomography of the abdominal revealed an obstructive, endoluminal gastric tumor: $13 \times 09 \times 12$ $\mathrm{cm}$, oval-shaped with regular contours, with low contract enhancement and gastric perforation associated with pneumoperitoneum (Figure-1). 

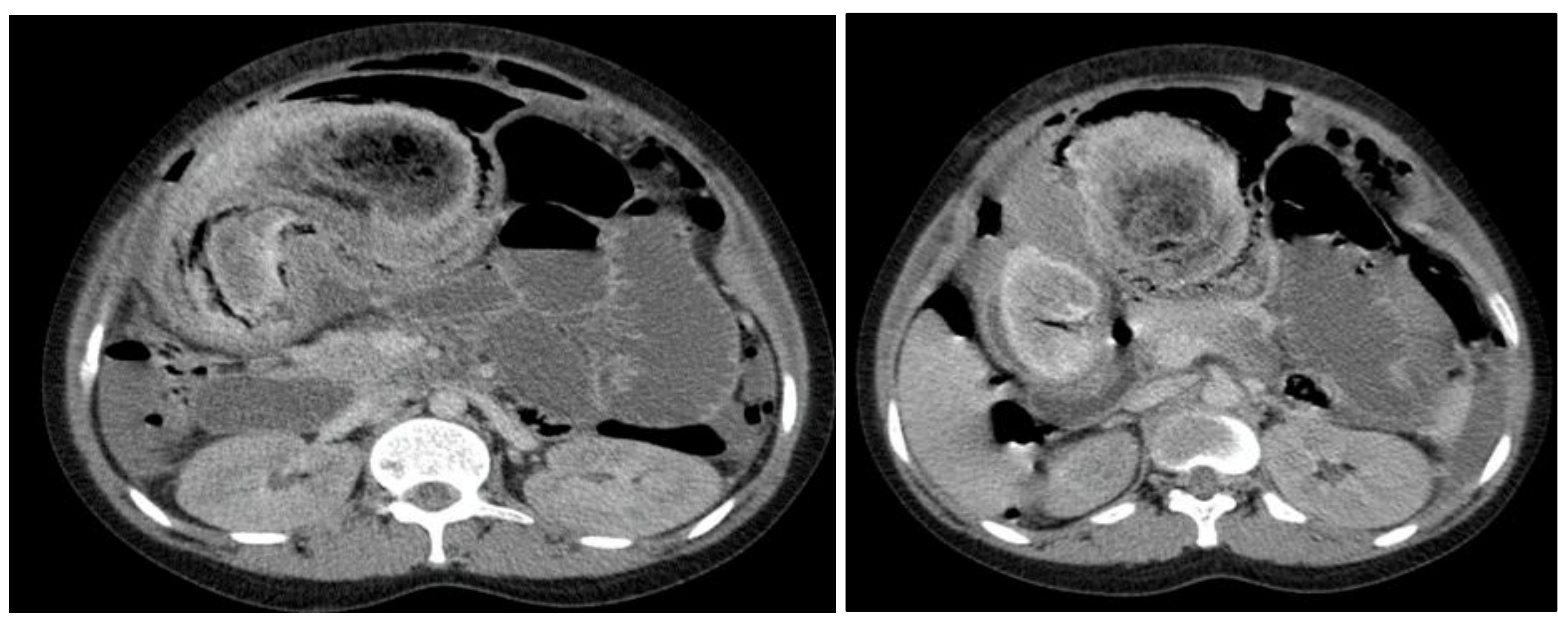

Fig-1: Scan image of gastric Trichobezoars complicated by peritonitis

Intravenous antibiotics were started and an emergency, and laparotomy was performed by the surgical team.

Intraoperatively, the stomach was noted to be enlarged by a firm, uniform mass. A large gastric perforation was located, high on the lesser curvature with gross peritoneal contamination. Aspiration of the effusion with sampling for bacteriological and cytological study. Total and complete extraction of the intraluminal mass was performed. The tumor was diagnosed as a trichobezoar (Figure-2).

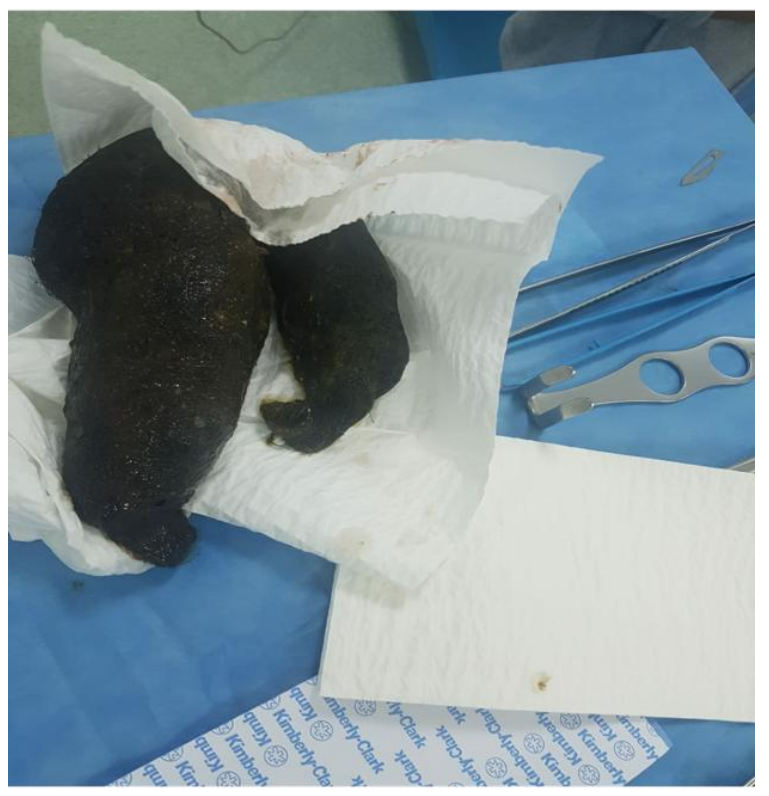

Fig-2: Gastric trichobizoar

Postoperatively, the patient recovered rapidly under the care of ours anesthesia team. a psychiatric opinion was required.

\section{DISCUSSION}

Gastric trichobezoar is a rare disease; secondary to an unusual accumulation, in the form of solid masses or concretions, of substances of all kinds in the gastrointestinal tract and especially in the stomach [5]. Despite a low prevalence, the mortality of gastric trichobezoar, if left untreated, can reach $30 \%$ due to complications [6].

The clinical presentation of gastric trichobezoar is highly variable, and complications may be a revealing feature [7]. These may include upper gastrointestinal bleeding due to parietal ulceration, mechanical gastric or bowel obstruction [8, 9], gastric or bowel perforation with peritonitis or subphrenic abscess [10], and digestive fistula [11].

Abdominal computed tomography confirms the diagnosis of complicated gastric trichobezoar [10], demonstrating two constant pathognomonic signs: the presence of tiny air bubbles dispersed in the mass and the absence of any attachment of the mass to the gastric wall [12].

Magnetic resonance imaging (MRI) also confirms the diagnosis [12].

Gastric trichobezoar in pregnancy is quite rare. A review of the literature identified three cases of uncomplicated gastric trichobezoar in pregnancy [12] and one case of complicated gastric perforation post partum [12].

Particularly in pregnant women and in the post partum period, it is necessary to inquire about strange eating habits during pregnancy, in order to facilitate the diagnosis. The management is always surgical in complicated trichobezoar, allowing exploration of the whole digestive tract, extraction of the gastric trichobezoar by gastrotomy, as well as extraction of any extension (tail) or fragment blocked at a distance from the stomach by one or several enterostomies [12].

The long-term evolution is often associated with the risk of intestinal complication related to the recurrence of trichobezoar [13]. 
The future of pregnancies is marked by the risk of intestinal obstruction, which remains rare, but can be associated with significant maternal and fetal morbidity [13].

\section{CONCLUSION}

Trichobezoar is a rare entity that usually presents insidiously with atypical and non-specific symptoms. Perforated gastric trichobezoar is a rare complication, especially in postpartum, the treatment is always surgical, with a risk of intestinal complications.

\section{REFERENCES}

1. Meyer-Rochow GY, Grunewald B. Laparoscopic Removal of a Gastric Trichobezoar in a Pregnant Woman. Surg Laparosc Endosc Percutan Tech. 2007 Apr;17(2):129-132.

2. L'aarje A, Elhattabi K, Lefryekh R, Fadil A, Khaiz D. Trichobézoard gastroduodénal et grêlique. Presse Med. 2015;45(2):265-269.

3. Gonuguntla V, Joshi DD. Rapunzel syndrome: a comprehensive review of an unusual case of trichobezoar. Clin Med Res. 2009 Sep;7(3): 99102.

4. Ibn Majdoub Hassani $\mathrm{K}$, El Bouhaddouti $\mathrm{H}$, Benamar Y, Mazaz1 K, Ait Taleb K. Trichobezoar gastrique - à propos de deux cas. Pan Afr Med J. 2010;6:19.

5. Mehta M, Patel RV. Intussusception and intestinal perforations caused by multiple trichobezoars. J
Pediatr Surg. 1992 Sep;27(9):1234-5.

6. Koc O, YIldIz FD, NarcI A, Sen TA. An unusual cause of gastric perforation in childhood: trichobezoar (Rapunzel syndrome). A case report. Eur J Pediatr. 2009 Apr;168(4):495-497.

7. Desplypere JP, Pract M, Verdonk G. An unusual case of trichobezoar: The Rapunzel Syndrome. Am J Gastroenterol. 1982 Jul;77(7):467-70.

8. Kaushik NK, Sharma YP, Negi A, Jaswal A. Images - Gastric trichobezoar. Ind J Radiol Imag. 1999;9(3):137-139.

9. Hafsa C, Golli M, Mekki M, Kriaa S. Trichobezoar géant chez l'enfant - Place de l'échographie et du transit oesogastroduodéna. J Pédiatrie Pueric. 2005;18(1):28-32

10. Siddiqi JA, El Daous A-M, Ahmed Shawosh YB. Trichobezoar due to psychiatric comorbidity: A rare case report. J Behav Health. 2017;6(1):1.

11. Benes J, Chmel J, Jodl J, Stuka C, Nevoral J. Treatment of a gastric bezoar by extracorporeal shock wave lithotripsy. Endoscopy. 1991 Nov;23(6):346-8.

12. Bharathan R, Datta $S$, Webb $P$, Griffin $S$. Trichobezoar and postpartum gastric perforation: A case report and brief review of literature. J Obstet Gynaecol. 2008;28(4):438-440

13. Ezziti1 M, Haddad F, Tahiri M, Hliwa W. Trichobézoard gastrique : à propos d'un cas. Pan Afr Med J. 2017 ; 26:74. 